\title{
EDITORIAL
}

Received: 2016-11-1

Accepted: 2016-11-3

\section{Chronic Pain and Cardiovascular Outcomes: Does Inflammation Play a Role?}

\author{
Ehsan Bahramali $\bowtie$ \\ Noncommunicable Diseases Research Center, Fasa University of Medical Sciences, Fasa, Iran
}

\section{Dear Reader,}

$\mathrm{C}$ hronic pain is currently considered a public health problem with high costs to the individual and society [1]. There are reports that distribution and intensity of pain are associated with cardiovascular (CV) risk [2]. Likewise, the relationship between pain and hypertension is potential of great pathophysiological and clinical interest [3,4]. Reverse causality can be proposed as well, in that common CV risk factors lead to atherosclerosis of the lumbar vessels and consequent low back pain (LBP) [5]. Besides there is a high prevalence of abdominal aortic aneurysm in patients with chronic LBP signifying the atherosclerotic complications as sources for otherwise unexplainable pain [6]. Other unfavorable vascular events like vascular dementia have also been reported to be associated with pain level in older adults and may have implications to the pathophysiologic consequences of prolonged pain in the brain [7].

To explain the proposed pathophysiological links between atherosclerosis and chronic pain, LBP is shown to be associated with an increased overall inflammatory response in human $[8,9]$. Dysregulation of the immune system has been nominated as a contributor to chronic pain. Even an enhanced innate immune response has been considered as a potential biomarker for the onset and perpetuation of chronic pain by some investigators [10]. The influence of chronic pain on the ini-

\section{GMJ}

o2016 Galen Medical Journal

Tel/Fax: +98 7136474503

PO Box 7193616563

Email:info@gmj.ir tiation of an overall inflammatory response in the body can give an explanation for the supposed link between pain and atherosclerosis. The pivotal role of inflammation, generally and in the vessel walls, is well understood in CV disease [11]. Inflammatory markers are increasingly used in clinical practice for risk stratification of patients with coronary artery disease and other atherosclerotic conditions. Atherosclerotic plaque formation, calcification, and rupture are induced and regulated by the local arterial inflammatory state, and this has gained serious implications in the determination of high-risk plaques with the help of novel techniques like optical coherence tomography during coronary angiography [12]. Meanwhile, screening for overall inflammatory response using measurements of serum high-sensitivity (hs)-CRP levels is now among the strongest predictors of $\mathrm{CV}$ risk and is widely used in preventive cardiology. In addition, among the major targets of statin therapy is a reduction in hs-CRP below $1 \mathrm{mg} / \mathrm{L}$ which underscores the principal role of inflammatory responses in increased $\mathrm{CV}$ risk [13].

Moreover, the neuroinflammatory mechanisms linking pain and psychobehavioral maladjustments with explicit indications in CV health have been described. Depression is triggered and aggravated by chronic pain which in turn affects CV outcomes [14]. Augmented sympathetic tone induced by pain has detrimental consequences for the CV health

\footnotetext{
Correspondence to:

Ehsan Bahrmali MD, Noncommunicable Diseases Research Center, Fasa University of Medical Sciences, Fasa, Iran

Telephone number: +98 715336300

Email Address: ebahramali@fums.ac.ir
} 
and commences a vicious circle in the central nervous system and peripheral vasculature [15]. The neurochemical pathways involved in the pain generation and perception thus have pathophysiological significance for the CV health and outcomes.

Available literature is inconclusive to identify the relation between chronic pain including LBP with CV disease and outcomes. With the high prevalence of LBP in different series (16$35 \%$ ), and with the negative consequences and links to atherosclerosis, chronic pain may be considered as a risk factor for $\mathrm{CV}$ disease in some populations [5]. Estimating the effect of such a risk factor for $\mathrm{CV}$ disease incidence and mortality at the population level may lead to preventive interventions to reduce the burden of the CV disease. Preventive strategies would theoretically be prescription of the painkillers with a variety of mechanisms of action to non-pharmacological remedies for chronic pain. Pertinent to these issues are the unfavorable $\mathrm{CV}$ outcomes associated with the use of analgesics and the subject of choosing the best preventive strategy for different populations based on the level of risk, origins of pain and comorbidities [16].

With the probable deleterious CV consequences of chronic pain and the adverse effects of painkillers at the same time, developing tools to accurately define the level of risk in an in- dividual with chronic pain is mandatory. Such risk estimation tools will theoretically apply to all patients suffering from chronic pain including LBP and serve as a potential measure to initiate medical therapy to reduce $\mathrm{CV}$ risk. In the current issue of Galen Medical Journal, Farjam et al. have presented a research protocol that involves a population survey of an area with around 40000 populations [ 17]. They have screened those between 35-70 years of age and determined the prevalence of LBP in this age group. A detailed questionnaire is filled out subsequently by trained personnel to look for a variety of risk factors and lifestyle determinants of LBP severity. This cohort is going to be followed for 10 years, and a biobank including serum, plasma, buffy coat and whole blood samples iscompiled. A cohort study like this is a very feasible means to investigate the long-term consequences of chronic pain on CV mortality and morbidity. The available biobank and repeated measurements as is described in the protocol will facilitate assays of inflammatory markers and will answer the question of whether there is a role for inflammation as the link between chronic pain and $\mathrm{CV}$ outcome.

[GMJ. 2016;5(4):162-64]

Keywords: Chronic Pain; Cardiovascular; Low Back Pain; Inflammation

References

1. Kurita GP, Sjøgren P, Juel K, Højsted J, Ekholm O. The burden of chronic pain: a cross-sectional survey focussing on diseases, immigration, and opioid use. Pain. 2012;153(12):2332-8.

2. Goodson NJ, Smith BH, Hocking LJ, McGilchrist MM, Dominiczak AF, Morris A, et al. Cardiovascular risk factors associated with the metabolic syndrome are more prevalent in people reporting chronic pain: results from a cross-sectional general population study. Pain. 2013;154(9):1595602.

3. Saccò M, Meschi M, Regolisti G, Detrenis S, Bianchi L, Bertorelli M, et al. The relationship between blood pressure and pain. J Clin Hypertens (Greenwich). 2013;15(8):600-5.

4. Olsen RB, Bruehl S, Nielsen CS, Rosseland LA, Eggen AE, Stubhaug A. Hypertension prevalence and diminished blood pressurerelated hypoalgesia in individuals reporting chronic pain in a general population: The Tromsø Study. Pain. 2013;154(2):257-62.

5. Ha IH, Lee J, Kim MR, Kim H, Shin JS. The association between the history of cardiovascular diseases and chronic low back pain in South Koreans: a cross-sectional study. PloS one. 2014;9(4):e93671.

6. Tsuchie H, Miyakoshi N, Kasukawa Y, Nishi T, Abe H, Takeshima M, et al. High prevalence of abdominal aortic aneurysm 
in patients with chronic low back pain. The Tohoku J Exp Med. 2013;230(2):83-6.

7. Scherder EJ, Plooij B, Achterberg WP, Pieper M, Wiegersma M, Lobbezoo F, et al. Chronic Pain in "Probable" Vascular Dementia: Preliminary Findings. Pain Med. 2015;16(3):442-50.

8. Saal JS. The role of inflammation in lumbar pain. Spine. 1995;20(16):1821-7.

9. Malik KM, Nelson A, Benzon H. Diseasemodifying Antirheumatic Drugs for the Treatment of Low Back Pain: A Systematic Review of the Literature. Pain Pract. 2015 May 1.

10. Generaal E, Vogelzangs N, Macfarlane GJ, Geenen R, Smit JH, Dekker J, Penninx BW. Basal inflammation and innate immune response in chronic multisite musculoskeletal pain. Pain. 2014;155(8):1605-12.

11. Libby P. Inflammation in cardiovascular disease. InCardiovascular Biomarkers 2006 (pp. 207-221). Humana Press.

12. Low AF, Tearney GJ, Bouma BE, Jang IK. Technology insight: optical coherence tomography-current status and future development. Nat Clin Pract Cardiovasc

Med. 2006;3(3):154-62.

13. Tsimikas S, Willerson JT, Ridker PM.

C-reactive protein and other emerging blood

biomarkers to optimize risk stratification of vulnerable patients. J Am Coll Cardiol. 2006;47(8s1):C19-31.

14. Burke NN, Finn DP, Roche M.

Neuroinflammatory Mechanisms Linking

Pain and Depression. Mod Trends

Pharmacopsychiatri. 2015;30:36-50.

15. Loeser JD, Melzack R. Pain: an overview. The Lancet. 1999;353(9164):1607-9.

16. Antman EM, Bennett JS, Daugherty A, Furberg C, Roberts H, Taubert KA. Use of nonsteroidal antiinflammatory drugs an update for clinicians: a scientific statement from the American Heart Association. Circulation. 2007;115(12):1634-42.

17. Farjam M, Askari A, Hoseinipour A, Homayounfar R, Jamshidi J, Khodabakhshi F, et al. A Cohort Study Protocol of Low Back Pain in Rural Area Inhabitants: Fasa Low Back Pain Cohort Study (FABPACS). GMJ.2016;5(4):225-29 